\title{
Differential expression of the novel oncogene, SALL4, in lymphoma, plasma cell myeloma, and acute lymphoblastic leukemia
}

\author{
Wei Cui ${ }^{1,2}$, Nikki R Kong ${ }^{1}$, Yupo Ma ${ }^{3}$, Hesham M Amin ${ }^{4}$, Raymond $\mathrm{Lai}^{5}$ and Li Chai ${ }^{1}$ \\ ${ }^{1}$ Department of Pathology, Joint Program in Transfusion Medicine, Brigham and Women's Hospital/ \\ Children's Hospital Boston, Harvard Medical School, Boston, MA, USA; ${ }^{2}$ Department of Clinical Laboratory, \\ Peking Union Medical College Hospital, Peking Union Medical College and Chinese Academy of Medical \\ Sciences, Wangfujing, Beijing, China; ${ }^{3}$ Division of Laboratory Medicine, Nevada Cancer Institute, Las Vegas, \\ NV, USA; ${ }^{4}$ Department of Hematopathology, University of Texas MD Anderson Cancer Center, Houston, TX, \\ USA and ${ }^{5}$ Department of Laboratory Medicine and Pathology, University of Alberta, Edmonton, Alberta, \\ Canada
}

\begin{abstract}
SALL4, a newly identified zinc-finger transcriptional factor important for embryonic development, is mapped to chromosome 20q13. Previously, we reported that SALL4 was constitutively expressed in acute myeloid leukemia and SALL4 transgenic mice developed acute myeloid leukemia. In this study, we aimed to survey SALL4 protein expression in benign and neoplastic hematopoietic tissues in addition to acute myeloid leukemia using immunostaining with a polyclonal anti-SALL4 antibody. Primary hematological tumors (178) and 15 benign hematopoietic tissues were examined. Reverse transcription-polymerase chain reaction was also performed to detect SALL4 mRNA expression on eight precursor B-cell lymphoblastic leukemia/lymphomas, 10 benign hematopoietic tissues, and seven hematopoietic cancer cell lines. Of the benign tissues, SALL4 expression was detectable only in CD34 ${ }^{+}$hematopoietic stem/progenitor cells (2/2 at protein level, $3 / 3$ at RNA level). In neoplastic tissues, only precursor B-cell lymphoblastic leukemia/lymphomas had detectable SALL4 (12/16 at protein level, 7/8 at RNA level), similar to that observed in acute myeloid leukemia. Of the seven cell lines examined, only those derived from acute myeloid leukemia and precursor B-cell lymphoblastic leukemia/ lymphomas were positive. To conclude, SALL4 expression is normally restricted to CD34+ hematopoietic stem/ progenitor cells. The persistence of SALL4 expression in leukemic blasts in precursor B-cell lymphoblastic leukemia/lymphomas resembles to what we observed in acute myeloid leukemia, and correlates with the maturation arrest of these cells. We have shown in our previous study that the constitutive expression of SALL4 in mice can lead to acute myeloid leukemia development. The similar expression pattern of SALL4 in acute myeloid leukemia and B-cell lymphoblastic leukemia/lymphomas suggests that these two disease entities may share similar biological features and/or mechanisms of leukemogenesis. More definite studies to investigate the role of SALL4 in the pathogenesis of B-cell lymphoblastic leukemia/lymphomas are needed in the future to address this question.
\end{abstract}

Modern Pathology (2006) 19, 1585-1592. doi:10.1038/modpathol.3800694; published online 22 September 2006

Keywords: SALL4; hematological malignancies; immunohistochemistry; reverse transcription-polymerase chain reaction

SALL1 through SALL4 in the SALL gene family are human homologs of Drosophila spalt (sal). ${ }^{1-4}$ In Drosophila, spalt is a homeotic gene that is essential

Correspondence: Dr L Chai, MD, Department of Pathology, Joint Program in Transfusion Medicine, Brigham and Women's Hospital/Children's Hospital Boston, Harvard Medical School, 75 Francis Street, Boston, MA 02115, USA.

E-mail: lchai@partners.org

Received 13 June 2006; revised 5 August 2006; accepted 16 August 2006; published online 22 September 2006 for the development of posterior head and anterior tail segments. It plays an important role in terminal differentiation of photoreceptors, tracheal development, ${ }^{5}$ and wing vein placement. ${ }^{6}$ Sal-related genes have been isolated from Caenorhabditis elegans, ${ }^{7}$ fish,${ }^{8,9}$ Xenopus, ${ }^{10,11}$ mice,${ }^{12,13}$ and humans. ${ }^{14}$ Each of these homologs is expressed during embryonic development and in specific adult tissues.

In humans, SALL1 is mutated in patients with Townes-Brocks syndrome, with phenotypes involving urogenital, limb, anal, and cardiac malforma- 
tions. ${ }^{15-19}$ Human SALL4 mutations are associated with Duane radial ray syndrome (or Okihiro syndrome)..$^{3,20-22}$ Duane radial ray syndrome is a human autosomal-dominant syndrome involving radialsided hand anomalies in association with Duane syndrome. Duane syndrome is characterized by an eye movement disorder (strabismus) in which eye abduction, or adduction, or both, is limited. Defects in hematopoiesis have not been reported to date in patients with Townes-Brocks syndrome or Duane radial ray syndrome. SALL3 is mapped to chromosome 18q23, and may be involved in the phenotype of patients with $18 \mathrm{q}$ deletion syndrome, which is characterized by developmental delay, midface hypoplasia, hearing loss, and tapered fingers. ${ }^{23}$ Unlike the other $S A L L$ gene family members, no known congenital malformations have been associated with SALL2 mutation. ${ }^{24}$ We have shown, however, that SALL2 is involved in normal hematopoiesis, while others have suggested that it is involved in tumorigenesis. ${ }^{25-28}$

We recently found that $S A L L 4$ expression is constitutively expressed in all cases of acute myeloid leukemia. ${ }^{29}$ To further understand the biological importance of this novel oncogene in other hematopoietic malignancies, we performed an analysis of SALL4 expression in normal and neoplastic hematopoietic tissues using both reverse transcription-polymerase chain reaction and immunostaining. We found a preferential expression of SALL4 in normal purified $\mathrm{CD}^{+} 4^{+}$hematopoietic stem/progenitor cells, leukemic blasts in precursor B-cell lymphoblastic leukemia/lymphomas, as well as acute myeloid leukemia. The biological importance of these findings is discussed.

\section{Materials and methods}

\section{Tissue and Tumor Samples}

Normal and neoplastic lymphoid tissue samples were randomly retrieved from the files of the Department of Laboratory Medicine and Pathology at the Cross Cancer Institute, the University Alberta, and the University of Arkansas. Two cases of normal $\mathrm{CD}^{+} 4^{+}$hematopoietic stem/progenitor cells were selected from mobilized peripheral blood of normal donors whose recipients died before the use of these cells, thus were considered discarded tissues. The other three cases were purchased from Cambrex Bio Science Walkersville Inc. (Walkersville, MD, USA). The use of these tissues has been approved by the Institutional Review Board. Normal tissue samples included benign reactive lymph nodes $(N=2)$, thymus $(N=2)$, spleen $(N=2)$, tonsils $(N=4)$, bone marrow aspirates $(N=2)$, bone marrow trephine biopsy $(N=3)$, and peripheral blood mononuclear cells $(N=5)$. Neoplastic tissue samples were derived from a total of 186 patients, and they included (1) malignant lymphomas $(N=113$ in total): non-Hodgkin's lymphoma $(N=108)$ with 42 T-cell and 66
B-cell; and Hodgkin's lymphoma $(N=5)$ with four classical and one lymphocyte predominant type; (2) lymphoblastic leukemia/lymphomas $(N=36)$, with 24 precursor B-cell and 12 precursor T-cell type; (3) plasma cell myelomas $(N=32)$; and (4) acute myeloid leukemias $(N=5)$, which served as positive controls. Within the group of B-cell lymphomas, there were follicular lymphomas $(N=7)$, small lymphocytic lymphomas $(N=30)$, marginal zone lymphomas $(N=9)$, mantle cell lymphomas $(N=5)$, diffuse large B-cell lymphomas $(N=10)$, and Burkitt's lymphomas $(N=5)$. Within the group of T-cell lymphomas, there were ALK-anaplastic large-cell lymphoma $(N=39)$, peripheral T-cell lymphoma $(N=2)$, and extranodal T/NK-cell lymphoma $(N=1)$. Diagnoses for all cases were based on morphological and immunophenotypic criteria according to the WHO classification system of hematopoietic neoplasms.

With a few exceptions, all of the tissue samples were formalin-fixed and paraffin-embedded, and they were subjected to immuohistochemical studies for SALL4 (described below). Exceptions were eight cases of precursor B-cell lymphoblastic leukemia/ lymphomas, all of which had $>70 \%$ blasts in the aspirates, two of the five bone marrow aspirates, all five samples of peripheral blood mononuclear cells, and all five samples of HSC/HPC, all of which were fresh tissue samples that were preserved in $10 \%$ dimethyl sulfoxide. All of the fresh samples of precursor B-cell lymphoblastic leukemia/lymphomas, bone marrow aspirates, peripheral blood mononuclear cells, as well as three of five cases of CD34 ${ }^{+}$ hematopoietic stem/progenitor cells were subjected to reverse transcription-polymerase chain reaction (method described below). The remaining two CD34 + hematopoietic stem/progenitor cells samples were subjected to immunocytochemical staining. Tissue microarrays incorporating triplicate cores of all cases of anaplastic large cell lymphoma, plasma cell myeloma, acute myeloid leukemia, and small lymphocytic lymphoma were used for immunohistochemistry. A manual tissue arrayer (Beecher Instruments, WI, USA) was used to construct the tissue arrays. Whole tissues were used for the remaining paraffin-embedded cases.

\section{Cell Lines and Cell Culture}

Each of the following seven ATCC cell lines was subjected to reverse transcription-polymerase chain reaction to detect SALL4 expression. A total of $1 \times 10^{6}$ cells were used for extraction of mRNA: RL and BJAB, derived from B-cell lymphoma; SUP-M2, derived from $\mathrm{ALK}^{+}$anaplastic large T-cell lymphoma; Reh, derived from precursor B-cell lymphoblastic leukemia/lymphomas; JURKAT, derived from Tcell lymphoblastic leukemia/lymphomas; MMIS, derived from plasma cell myeloma; and Kasumi-1, derived from acute myeloid leukemia. All of these 
cell lines were stored in liquid nitrogen and cultured in RPMI 1640 supplemented with 10\% heat-inactivated fetal calf serum, $2 \mathrm{mmol} / \mathrm{l}$ glutamine, $100 \mu \mathrm{g} / \mathrm{ml}$ streptomycin, and $100 \mathrm{U} / \mathrm{ml}$ penicillin.

\section{Magnetic Nanoparticles for Selecting Normal CD34 ${ }^{+}$ Hematopoietic Stem/Progenitor Cells and Flow Cytometry for Assessing Purity and Viability}

To examine the expression of SALL4 gene and protein in normal $\mathrm{CD}^{+} 4^{+}$hematopoietic stem/ progenitor cells, magnetic nanoparticles (StemCell Technologies, Vancouver, BC, Canada) was used to positively select CD $34^{+}$cells from previously frozen normal mobilized peripheral blood.

Frozen cells were thawed at $37^{\circ} \mathrm{C}$ in the water bath. Mononuclear cells were prepared using Ficoll density separation (StemCell Technologies), and then washed for $5 \mathrm{~min}$ in PBS containing $2 \% \mathrm{FBS}$ and $1 \mathrm{mM}$ EDTA (StemCell Technologies). Clumpy suspension was filtered through a $70 \mu \mathrm{m}$ mesh nylon strainer (BD Biosciences, San Diego, CA, USA). Mononuclear cell suspensions at a concentration of $1 \times 10^{8} \mathrm{cells} / \mathrm{ml}$ were placed in $12 \times 75 \mathrm{~mm}$ polystyrene tube to properly fit into the Magnet rack. CD34 antibody (clone QBend 10) was then added, and magnetic selection was completed according to the manufacturer's instructions (StemCell Technologies).

Purity of the positively selected cells was assessed using phycoerythrin (PE)-conjugated CD34 antibody (clone 8G12, BD Biosciences), and cell viability was estimated using propidium iodide (PI, BD Biosciences) by flow cytometry.

\section{Reverse Transcription-Polymerase Chain Reaction for Detecting SALL4 Expression}

To examine the expression of SALL4 gene at the mRNA level, reverse transcription-polymerase chain reaction was performed with cells harvested from the cell lines. The QuickPrep ${ }^{\mathrm{TM}}$ Micro mRNA purification kit (GE Healthcare, Boston, MA, USA) was used to extract mRNA. First-strand cDNA was synthesized with the use of bulk first-strand cDNA mix and random primer (GE Healthcare, Boston, MA, USA). PCR amplification using cDNA was performed according to the manufacturer's instructions (Qiagen, Boston, MA, USA). Oligonucleotides were synthesized as specific primers for SALL4 by Integrated DNA Technologies Inc. (IA, Boston, MA, USA). Detection of $\beta$-actin was used as a control reaction in each run.

The sequences of primers were as follow: SALL4 forward primer: 5'-CATGATGGCTTCCTTAGATGC CCCAG-3', reverse primer: 5'-CCGTGTGTCATGT AGTGAACCTTTAAG- $3^{\prime}$. $\beta$-Actin forward primer: 5'-GCACCCAGCACAATGAAG-3', reverse primer: $5^{\prime}$-ACTCGTCATACTCCTGCTTG- ${ }^{\prime}$.
The expected sizes of the PCR products were approximately $500 \mathrm{bp}$ for $S A L L 4$ and $120 \mathrm{bp}$ for $\beta$ actin. PCR $50 \mathrm{bp}$ low ladder (Sigma-Aldrich, St Louis, MO, USA) was run in parallel as a molecular size marker.

\section{Immunostaining for SALL4}

To examine the SALL4 protein expression, immunostaining was performed according to standard techniques, which are briefly described here. Formalin-fixed, paraffin-embedded tissue sections of $5 \mu \mathrm{m}$ thickness were de-paraffinized and hydrated. Heat-induced epitope was retrieved with the use of citrate buffer (pH 6; ZYMED Laboratories Inc., South San Francisco, CA, USA) and a pressure cooker (Biocare Medical, Concord, CA, USA). After incubation for $2 \mathrm{~min}$ at $100^{\circ} \mathrm{C}$, slides were cooled for $25 \mathrm{~min}$, rinsed in running tap water, and then

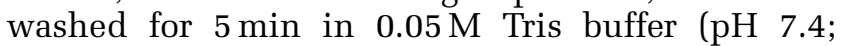
Sigma-Aldrich) with $0.05 \%$ Tween-20 (DakoCytomation, Carpinteria, CA, USA). Endogenous peroxidase was then blocked by incubating the tissue sections in $\mathrm{H}_{2} \mathrm{O}_{2}$ for 5 min (DakoCytomation). Subsequently, the tissue sections were incubated for $1 \mathrm{~h}$ with the anti-SALL4 antibody (1:500) in a humidified chamber at room temperature. ${ }^{29}$ After three washes with Tris buffer, tissue sections were incubated with Envision anti-rabbit IgG (DakoCytomation) for $30 \mathrm{~min}$ at room temperature. After additional three washes, tissue sections were incubated with $250 \mu \mathrm{l}$ of chromogen substrate (DakoCytomation) for $10 \mathrm{~min}$ or until color development. Hematoxylin (Fisher Scientific, Boston, MA, USA) was used as a counterstain. The immunostaining protocol for two fresh samples of hematopoietic stem/progenitor cells was essentially similar, except that the cytospin preparations were fixed in cold acetone $\left(-20^{\circ} \mathrm{C}\right.$; Fisher Scientific) for $4 \mathrm{~min}$ at room temperature and then air-dried for $30 \mathrm{~min}$ prior to the immunostaining.

In each case, immunostaining was considered to be positive for SALL4 when more than $15 \%$ of cells showed definitive nuclear staining. Acute myeloid leukemia was chosen as the positive control in this study on the basis of our previous survey of 81 acute myeloid leukemia cases; nuclear staining for SALL4 protein was present in $100 \%$ of acute myeloid leukemia blasts.

\section{Results}

\section{Detection of SALL4 Protein Expression by Immunostaining in Benign and Neoplastic Hematopoietic Cells}

Benign $\mathrm{CD}_{3} 4^{+}$hematopoietic stem/progenitor cells, normal bone marrow aspirates, and leukemia samples

We first examined $S A L L 4$ protein expression during normal hematopoietic differentiation (CD34 ${ }^{+}$ 
hematopoietic stem/progenitor cells and whole bone marrows), and its malignant counterpart, leukemia samples. As illustrated in Figure 1, immunostaining revealed that the SALL4 protein was expressed in the nuclei of normal CD34 ${ }^{+}$ hematopoietic stem/progenitor cells $(N=2)$ and those of precursor B-cell lymphoblastic leukemia/ lymphomas blasts $(N=16,75 \%)$, and the staining pattern was similar to that observed in the positive control, acute myeloid leukemic blasts. In contrast, SALL4 protein expression was negative in all precursor T-cell lymphoblastic leukemia/lymphomas $(N=12$, Figure $1 \mathrm{~d})$. Normal whole bone marrow trephine biopsy $(N=3)$ samples were also negative for SALL4 by immunohistochemistry. Positive cases were defined as $>15 \%$ of immunostained cells with only nuclear staining. In normal whole bone marrow, $<5 \%$ cells were observed to be positive, which were probably the $\mathrm{CD}_{3} 4^{+}$hematopoietic stem/progenitor cells. Faint cytoplasmic staining was observed in occasional cases, but this was regarded as nonspecific and only definitive nuclear staining was considered to be positive. Nonhematopoietic cells such as endothelial cells were negative.

\section{Benign lymphoid tissues}

We then studied SALL4 protein expression in benign mature lymphoid tissues. By immunohistochemistry, SALL4 protein expression was considered to be negative in 10 samples of normal lymphoid tissues examined, including two benign lymph nodes, four reactive tonsils, two benign thymus (surgically removed for cardiac operation), and two benign spleens (surgically removed due to trauma) (Figure 2). There were none or rare $(<1 \%)$ positive cells detected in these samples.

\section{Malignant lymphomas and plasma cell myeloma}

We next investigated SALL4 expression in the malignant counterpart of the benign lymphoid tissues, such as lymphoma and plasma cell myeloma. As illustrated in Figure 3 and summarized in
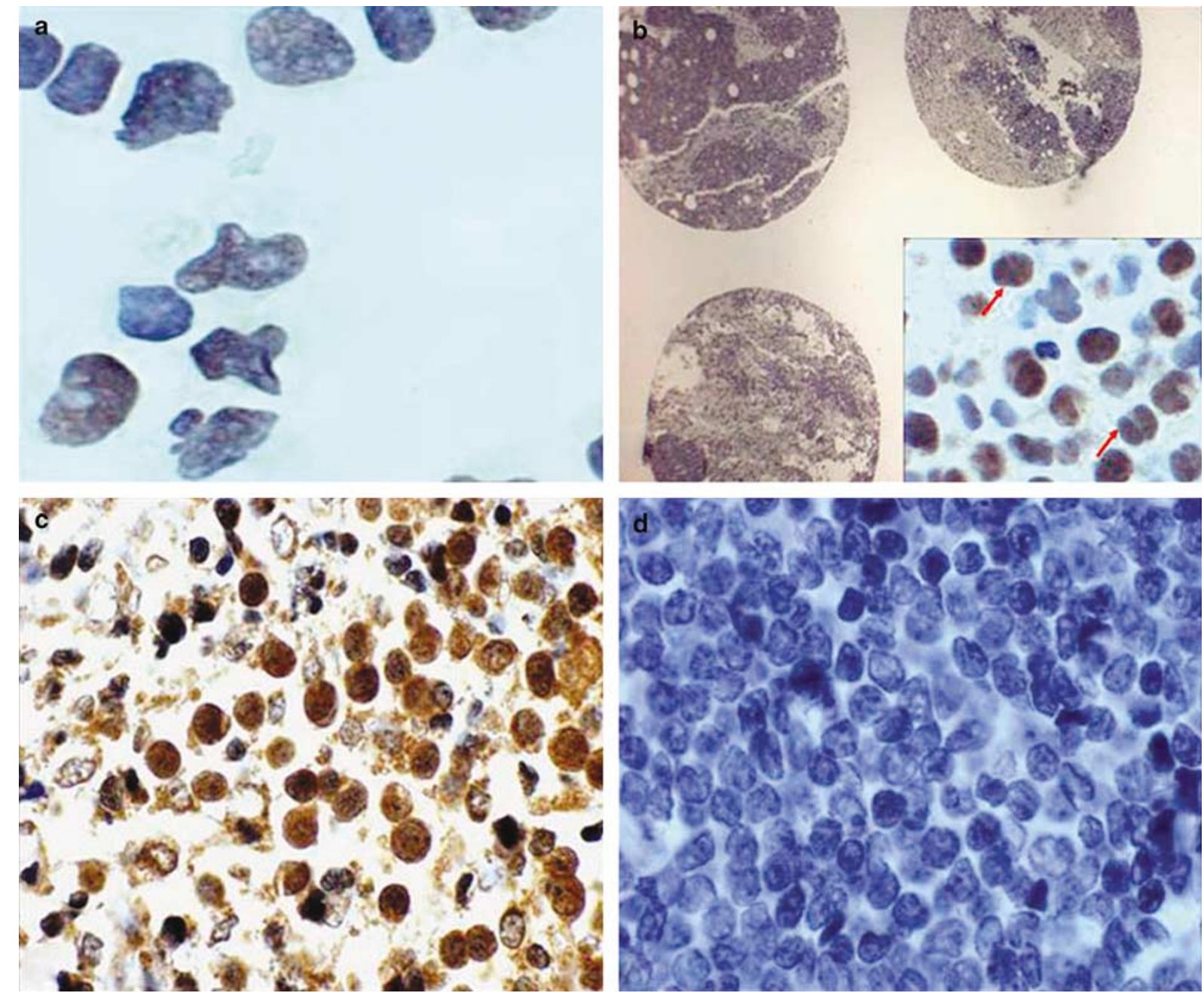

Figure 1 SALL4 protein expression in normal CD34 ${ }^{+}$hematopoietic stem/progenitor cells and lymphoblastic leukemia/lymphomas (immunoperoxidase). SALL4 was positive in CD $34^{+}$hematopoietic stem/progenitor cells $(\mathbf{a}, \times 600)$ and B-cell lymphoblastic leukemia/ lymphomas blasts $(\mathbf{c}, \times 400)$, identical to the positive control $(\mathrm{AML})(\mathbf{b}, \times 400)$, but negative in T-cell lymphoblastic leukemia/ lymphomas $(d, \times 400)$. 
Table 1, SALL4 protein expression was negative in all of the 113 B- or T-cell lymphoma and 32 plasma cell myeloma samples examined.

\section{Detection of SALL4 mRNA Expression in Normal Peripheral Blood Mononuclear Cells, Benign Bone Marrow Aspirates, Acute Lymphoblastic Leukemia, and Hematopoietic Cancer Cell Lines}

To correlate with the findings on its protein expression, we next examined the mRNA expression of SALL4 at different stages of normal hematopoietic differentiation, in primary leukemic samples and hematopoietic cancer cell lines. Summarized in
Table 1 and illustrated in Figure 4, SALL4 mRNA was expressed in normal CD34 ${ }^{+}$hematopoietic stem/progenitor cells $\left(N=3\right.$, purity of normal CD34 ${ }^{+}$ hematopoietic stem/progenitor cells is 95, 95, and $98 \%$ with cell viability is 99, 99, and 97\%, respectively, for each sample); it was down regulated in benign bone marrow aspirates $(N=2)$ as $S A L L 4$ mRNA was barely detectable, probably due to its $<5 \%$ components of CD $34^{+}$hematopoietic stem/ progenitor cells. No detectable mRNA expression for $S A L L 4$ was identified in normal peripheral blood $(N=5)$. These results were consistent with our previous reports on $S A L L 4$ mRNA expression during normal hematopoiesis using real-time reverse transcription-polymerase chain reaction method. ${ }^{29}$
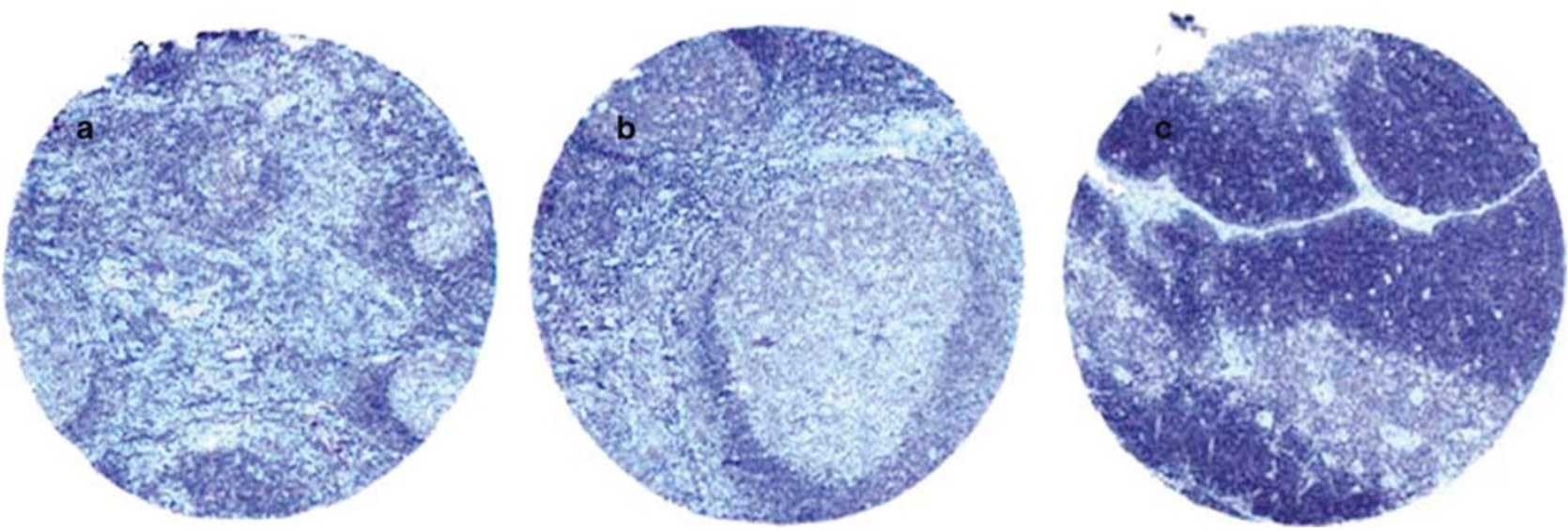

Figure 2 Immunohistochemical staining for SALL4 in normal lymph tissues. No detectable immunostaining (immunoperoxidase) of SALL4 protein was seen in benign lymph nodes $(\mathbf{a}, \times 10)$, normal tonsil $(\mathbf{b}, \times 10)$ and normal thymus $(\mathbf{c}, \times 10)$. All staining of $\mathrm{T}$ zones, mantle zones, germinal centers, and splenic marginal zones was negative.
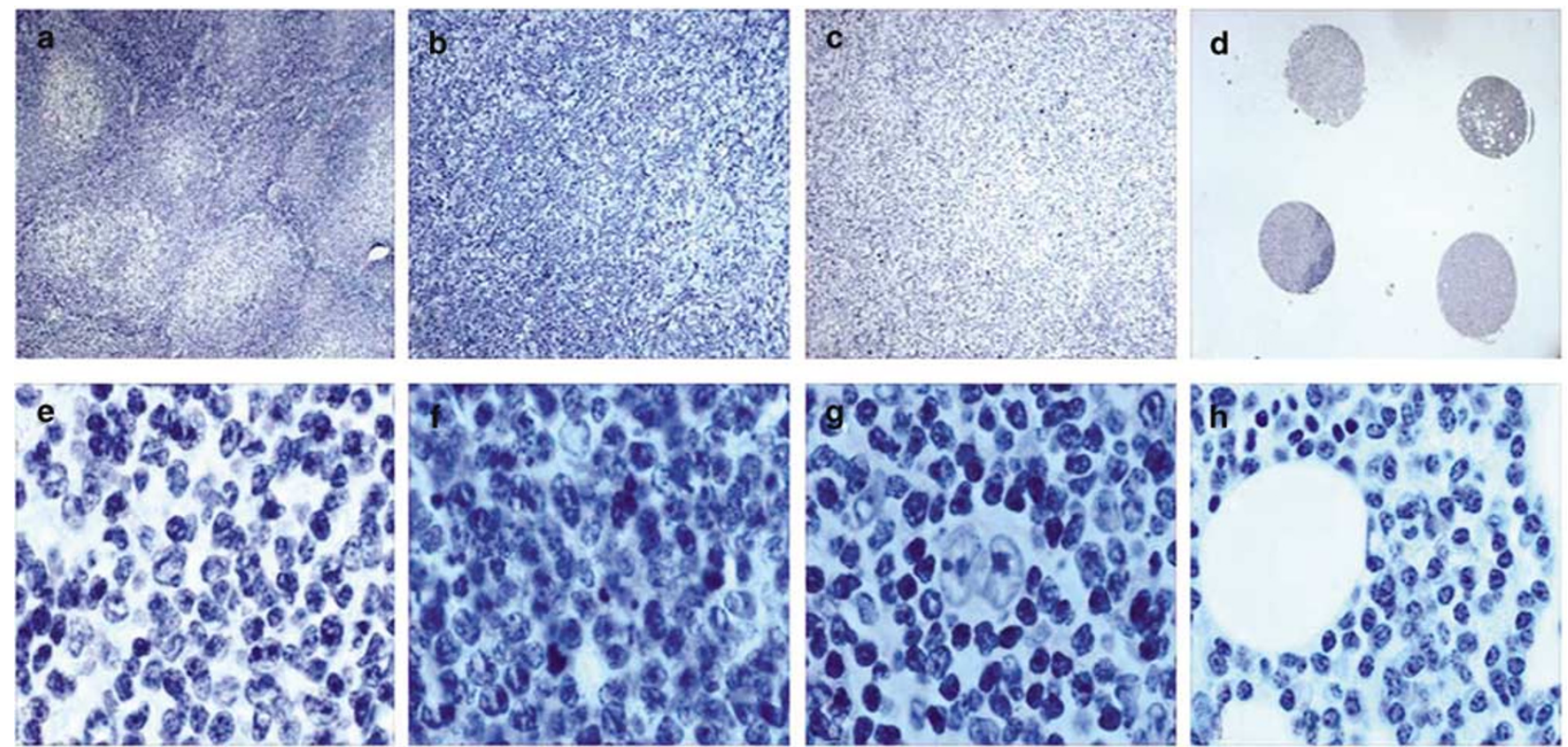

Figure 3 Immunohistochemical staining for SALL4 in lymphoma and plasma cell myeloma (immunoperoxidase). SALL4 protein was negative in follicular lymphoma $(\mathbf{a}, \times 10 ; \mathbf{e}, \times 600)$, Burkitt's lymphoma $(\mathbf{b}, \times 10 ; \mathbf{f}, \times 600)$, Hodgkin's lymphoma $(\mathbf{c}, \times 10 ; \mathbf{g}, \times 600)$, and plasma cell myeloma $(\mathbf{d}, \times 1 ; \mathbf{h}, \times 600)$. 
Table 1 SALL4 expression in normal hematopoietic tissues, lymphoma, plasma cell myeloma and acute lymphoblastic leukemia

\begin{tabular}{|c|c|c|}
\hline Primary tumor and normal tissue samples & Detection method (IHC, RT-PCR) & $\begin{array}{c}\text { No. of SALL4-positive cases per total no. of } \\
\text { cases examined (percentage) }\end{array}$ \\
\hline Non-Hodgkin’s lymphoma & IHC & $0 / 108$ \\
\hline Hodgkin’s lymphoma & IHC & $0 / 5$ \\
\hline Plasma cell myeloma & IHC & $0 / 32$ \\
\hline $\begin{array}{l}\text { Precursor T-cell acute lymphoblastic } \\
\text { leukemia/lymphoma }\end{array}$ & IHC & $0 / 12$ \\
\hline $\begin{array}{l}\text { Precursor B-cell acute lymphoblastic } \\
\text { leukemia/lymphoma }\end{array}$ & RT-PCR $(N=8)$, IHC $(N=16)$ & $19 / 24(79.1 \%)^{b}$ \\
\hline Positive control (AML) & IHC & $5 / 5(100 \%)$ \\
\hline Normal CD34 ${ }^{+}$HSC/HPC cells & $\begin{array}{l}\text { Immunocytochemistry }(N=2) \text {; RT-PCR } \\
(N=3)\end{array}$ & $5 / 5(100 \%)$ \\
\hline Normal benign reactive lymphoid tissue & IHC & $0 / 10$ \\
\hline Normal bone marrow & $\begin{array}{l}\text { Trepine biopsy assessed by IHC }(N=3) \text {, } \\
\text { aspirates assessed by RT-PCR }(N=2)\end{array}$ & $0 / 5$ \\
\hline $\begin{array}{l}\text { Normal peripheral blood } \\
\text { Total }\end{array}$ & RT-PCR $(N=5)$ & $\begin{array}{c}0 / 5 \\
29 / 211(13.7 \%)\end{array}$ \\
\hline
\end{tabular}

RT-PCR $=$ reverse transcriptase-polymerase chain reaction; IHC $=$ immunohistochemistry.

${ }^{\mathrm{a}}$ Criteria for positive expression of SALL4 were the presence of only nuclear staining and staining of more than $15 \%$ of cells.

${ }^{\mathrm{b}}$ In precursor B-cell lymphoblastic leukemia/lymphoma, seven of eight cases are positive for $S A L L 4$ gene mRNA expression (7/8, $87.5 \%$ ); 12 of 16 cases are positive for $S A L L 4$ protein expression (12/16, 75\%).

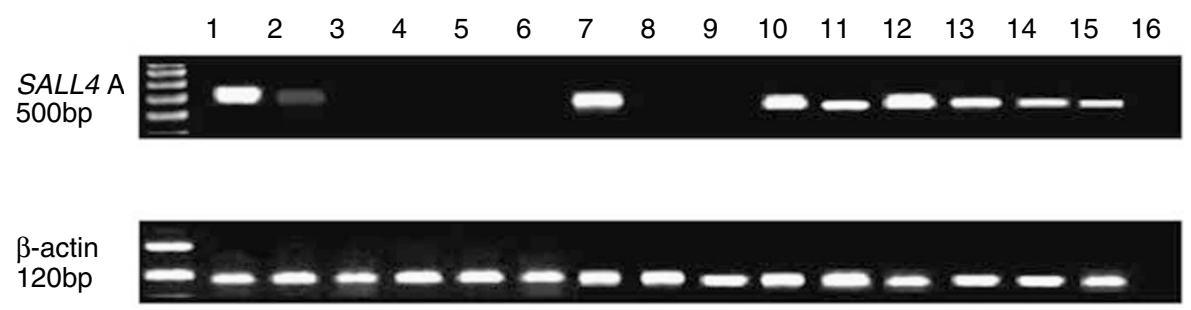

Figure $4 S A L L 4$ mRNA expression obtained from reverse transcription-polymerase chain reaction assay in normal hematopoietic tissues, primary B-cell lymphoblastic leukemia/lymphomas and cell lines from hematological malignancies. SALL4 mRNA was detected in normal CD34 ${ }^{+}$hematopoietic stem/progenitor cells (lane 1), in precursor B-cell cell line Reh (lane 7), acute myeloid leukemia M2 cell line Kasumi (lane 10), and primary B-cell lymphoblastic leukemia/lymphomas bone marrow (lanes 11-15). SALL4 mRNA was barely detected in normal whole bone marrow (lane 2). Negative results included: normal peripheral blood (lane 3), Burkitt's pre-B-cell lymphoma cell line BJAB (lane 4), B-cell lymphoblastic lymphoma cell line RL (lane 5), T-cell lymphoma cell line SUP-M2 (lane 6), T-cell lymphoblastic leukemia/lymphomas cell line JURKAT (lane 8), plasma cell myeloma cell line MMIS (lane 9), and negative control (lane 16). $\beta$-Actin was used as an internal control after 35 cycles.

Similar to our findings on SALL4 protein expression, seven of eight B-cell lymphoblastic leukemia/ lymphomas samples examined were positive for $S A L L 4$. Of the cell lines, only those of precursor Bcell lymphoblastic leukemia/lymphomas (Reh-1) and acute myeloid leukemia (Kasumi-1) were positive for $S A L L 4$. SALL4 mRNA was negative in all of the lymphoma cell lines (RL, BJAB, and SUP-M2), those derived from the precursor T-cell lymphoblastic leukemia/lymphomas (JURKET), and plasma cell myeloma (MMIS).

\section{Discussion}

We recently identified that $S A L L 4$, a gene important for embryonic development, is constitutively expressed in human acute myeloid leukemia, and its overexpression in the transgenic mouse model leads to generation of acute myeloid leukemia. ${ }^{29}$ These findings strongly implicate that SALL4 is an oncogene which is involved in myeloid leukemogenesis. In order to further understand the biological significance of SALL4, particularly in the lymphoid tissues, we performed a survey on a broad range of normal and neoplastic hematopoietic cells. We found that the expression of the SALL4 gene is restricted to the CD34 ${ }^{+}$hematopoietic stem/progenitor cells during normal hematopoiesis, and not in normal mature hematopoietic cells and benign reactive lymphoid tissues. Among the malignant counterparts, we only detected SALL4 expression in acute myeloid leukemia and large portion of precursor B-cell lymphoblastic leukemia/lymphomas patient samples. Our studies of a series of hematopoietic cancer cell lines also revealed the same SALL4 expression pattern, with SALL4 expression detectable only in cell lines derived from acute myeloid leukemia and precursor B-cell lymphoblastic leukemia/lymphomas. 
Like other $S A L L$ genes, $S A L L 4$ plays an important role in human development, with heterozygous mutations of SALL4 leading to Duane radial ray syndrome. ${ }^{16,17,21}$ Its role in hematopoiesis is not completely understood. As revealed in this study, SALL4 is only expressed in CD34 ${ }^{+}$hematopoietic stem/progenitor cells, but not mature myeloid or lymphoid cells. Thus, SALL4 expression is downregulated in normal maturation and differentiation in both myeloid and lymphoid cell lineages. This observation is in contrast with the expression of SALL4 in most cases of acute myeloid leukemia and precursor B-cell lymphoblastic leukemia/lymphomas, in which SALL4 is constitutively expressed at the mRNA and protein levels. Thus, the failure of downregulation of SALL4 in acute myeloid leukemia and precursor B-cell lymphoblastic leukemia/ lymphomas correlates with the maturation arrest in the neoplastic blast population in these leukemias. We have already shown that the overexpression of SALL4 in mice can lead to acute myeloid leukemia development in about $50 \%$ transgenic mice. In future studies, it will be of great interest to examine the remaining SALL4 transgenic mice for the potential B-cell lymphoblastic leukemia/lymphomas development.

While our findings suggest that SALL4 might play roles in leukemogenesis of precursor B-cell lymphoblastic leukemia/lymphomas, this novel oncogene is not expressed in precursor T-cell lymphoblastic leukemia/lymphomas. These findings suggest that the pathogenesis of precursor B-cell lymphoblastic leukemia/lymphomas is different from that of precursor T-cell lymphoblastic leukemia/lymphomas. Also in parallel with these findings, the vast majority of the chronic myeloid leukemia cases that transform into acute leukemia are rarely T-cell lymphoblastic leukemia/lymphomas, but rather acute myeloid leukemia or precursor B-cell lymphoblastic leukemia/lymphomas. These observations may be explained by a common pathway shared by the leukemogenesis of precursor B-cell lymphoblastic leukemia/lymphomas and acute myeloid leukemia. Recently, a B-cell/myeloid common progenitor that gives rise to both $\mathrm{B}$ and myeloid lineages has been identified as a population $\mathrm{CD}_{3} 4^{+} \mathrm{CD} 19^{+}$ $\mathrm{CXCR}^{-}$in humans and $\mathrm{C}^{-} \mathrm{Kit}^{+} \mathrm{B} 220^{+} \mathrm{CD} 19^{-}$in mice. $^{30,31}$ Additional evidence supporting the existence of a B-cell/myeloid common progenitor comes from a functional study of the transcription factor PU.1 in hematopoiesis. ${ }^{32,33}$ When PU.1 was homozygously deleted in a murine model, no B cells, macrophages, or granulocytes could be detected, and the animals were anemic. ${ }^{32}$ One explanation for this observed phenotype in PU.1knockout mice is that PU.1 is essential to the differentiation of B-cell and myeloid lineages, but not that of T-cells. Therefore, on the basis of SALL4's similar expression patterns in acute myeloid leukemia and B-cell lymphoblastic leukemia/lymphomas, we hypothesize that SALL4 (in analogy to PU.1) is required for the development of the B-cell/myeloid common progenitor, and dysregulation of its expression can lead to maturation arrest of these cells and predisposing to leukemogenesis of acute myeloid leukemia and precursor B-cell lymphoblastic leukemia/lymphomas. While the precise stage position of B-cell/myeloid common progenitor population needs further definition within the hematopoietic paradigm, the identification of these B/myeloid common progenitors support the concept that the development of the B-cell and the myeloid lineages is more closely related than that of either cell type with the T-cell development.

While our data may implicate the role of SALL4 in leukemogenesis of precursor B-cell lymphoblastic leukemia/lymphomas, a small subset of these cases had no evidence of SALL4 expression, either at the mRNA or protein levels. These findings suggest the pathogenesis of precursor B-cell lymphoblastic leukemia/lymphomas is heterogeneous. Larger studies with more cases will be required to compare $S A L L 4$-positive and SALL4-negative cases, in terms of morphology, cytogenetics, patient age, and clinical outcome.

Lastly, our data suggest that SALL4 may be diagnostically useful due to its high specificity to acute myeloid leukemia and precursor B-cell lymphoblastic leukemia/lymphomas. Similar to terminal deoxynucleotidase, SALL4 appears to be a marker for immaturity and thus is useful in distinguishing precursor B-cell lymphoblastic leukemia/lymphomas from other aggressive B-cell lymphoma such as Burkitt's lymphoma.

\section{Acknowledgements}

This work was supported in part by the NIH under Grants K08 CA097185 and P20 RR016464 (to YM); and K08 DK063220 and National Blood Foundation (to LC).

\section{References}

1 Kohlhase J, Schuh R, Dowe G, et al. Isolation, characterization, and organ-specific expression of two novel human zinc finger genes related to the Drosophila gene spalt. Genomics 1996;38:291-298.

2 Kohlhase J, Hausmann S, Stoimenovic G, et al. SALL3, a new member of the human spalt-like gene family, maps to 18q23. Genomics 1999;62:216-222.

3 Al-Baradie R, Yamada K, St Hilaire C, et al. Duane radial ray syndrome (Okihiro syndrome) maps to $20 \mathrm{q} 13$ and results from mutations in $S A L L 4$, a new member of the SAL family. Am J Hum Genet 2002;71:1195-1199.

4 Kuhnlein RP, Frommer G, Friedrich M, et al. Spalt encodes an evolutionarily conserved zinc finger protein of novel structure which provides homeotic gene function in the head and tail region of the Drosophila embryo. EMBO J 1994;13:168-179. 
5 Boube M, Llimargas M, Casanova J. Cross-regulatory interactions among tracheal genes support a cooperative model for the induction of tracheal fates in the Drosophila embryo. Mech Develop 2000;91: 271-278.

6 Mollereau B, Dominguez M, Webel R, et al. Two-step process for photoreceptor formation in Drosophila. Nature 2001;412:911-913.

7 Basson M, Horvitz HR. The Caenorhabditis elegans gene sem-4 controls neuronal and mesodermal cell development and encodes a zinc finger protein. Gene Dev 1996;10:1953-1965.

8 Camp E, Hope R, Kortschak RD, et al. Expression of three spalt (sal) gene homologues in zebrafish embryos. Dev Genes Evol 2003;213:35-43.

9 Koster R, Stick R, Loosli F, et al. Medaka spalt acts as a target gene of hedgehog signaling. Development 1997;124:3147-3156.

10 Hollemann T, Schuh R, Pieler T, et al. Xenopus Xsal-1, a vertebrate homolog of the region specific homeotic gene spalt of Drosophila. Mech Develop 1996;55: 19-32.

11 Neff AW, King MW, Harty MW, et al. Expression of Xenopus XlSALL4 during limb development and regeneration. Dev Dynam 2005;233:356-367.

12 Ott T, Parrish M, Bond K, et al. A new member of the spalt like zinc finger protein family, Msal-3, is expressed in the CNS and sites of epithelial/mesenchymal interaction. Mech Develop 2001;101:203-207.

13 Kohlhase J, Altmann M, Archangelo L, et al. Genomic cloning, chromosomal mapping, and expression analysis of msal-2. Mamm Genome 2000;11:64-68.

14 Marlin S, Blanchard S, Slim R, et al. Townes-Brocks syndrome: detection of a SALL1 mutation hot spot and evidence for a position effect in one patient. Hum Mutat 1999;14:377-386.

15 Dong PD, Todi SV, Eberl DF, et al. Drosophila spalt/ spalt-related mutants exhibit Townes-Brocks' syndrome phenotypes. Proc Natl Acad Sci USA 2003;100: 10293-10298.

16 Nishinakamura R, Matsumoto Y, Nakamura K, et al. Murine homolog of SALL1 is essential for ureteric bud invasion in kidney development. Development 2001;128:3105-3115.

17 Ma Y, Chai L, Cortez SC, et al. SALL1 expression in the human pituitary-adrenal/gonadal axis. J Endocrinol 2002;173:437-448.

18 Ma Y, Singer DB, Gozman A, et al. Hsal 1 is related to kidney and gonad development and is expressed in Wilms tumor. Pediatr Nephrol 2001;16:701-709.

19 Kohlhase J, Heinrich M, Schubert L, et al. Okihiro syndrome is caused by SALL4 mutations. Hum Mol Genet 2002;11:2979-2987.

20 Borozdin W, Wright MJ, Hennekam RC, et al. Novel mutations in the gene SALL4 provide further evidence for acro-renal-ocular and Okihiro syndromes being allelic entities, and extend the phenotypic spectrum. J Med Genet 2004;41:e102.

21 Kohlhase J, Schubert L, Liebers M, et al. Mutations at the $S A L L 4$ locus on chromosome 20 result in a range of clinically overlapping phenotypes, including Okihiro syndrome, Holt-Oram syndrome, acro-renal-ocular syndrome, and patients previously reported to represent thalidomide embryopathy. J Med Genet 2003;40: 473-478.

22 Parrish M, Ott T, Lance-Jones C, et al. Loss of the Sall3 gene leads to palate deficiency, abnormalities in cranial nerves, and perinatal lethality. Mol Cell Biol 2004;24:7102-7112.

23 Sato A, Matsumoto Y, Koide U, et al. Zinc finger protein sall2 is not essential for embryonic and kidney development. Mol Cell Biol 2003;23:62-69.

$24 \mathrm{Li} \mathrm{D,} \mathrm{Tian} \mathrm{Y,} \mathrm{Ma} \mathrm{Y,} \mathrm{et} \mathrm{al.} \mathrm{p150} \mathrm{(Sal2)} \mathrm{is} \mathrm{a} \mathrm{p53-}$ independent regulator of p21(WAF1/CIP). Mol Cell Biol 2004;24:3885-3893.

25 Ma Y, Li D, Chai L, et al. Cloning and characterization of two promoters for the human HSAL2 gene and their transcriptional repression by the Wilms tumor suppressor gene product. J Biol Chem 2001;276: 48223-48230.

26 Nielsen TO, Hsu FD, O’Connell JX, et al. Tissue microarray validation of epidermal growth factor receptor and SALL2 in synovial sarcoma with comparison to tumors of similar histology. Am J Pathol 2003; 163:1449-1456.

27 Li CM, Guo M, Borczuk A, et al. Gene expression in Wilms' tumor mimics the earliest committed stage in the metanephric mesenchymal-epithelial transition. Am J Pathol 2002;160:2181-2190.

$28 \mathrm{Li} \mathrm{D}$, Dower K, Ma Y, et al. A tumor host range selection procedure identifies p150(sal2) as a target of polyoma virus large T antigen. Proc Natl Acad Sci USA 2001;98:14619-14624.

29 Ma Y, Cui W, Yang J, et al. SALL4, a novel oncogene, is constitutively expressed in human acute myeloid leukemia (AML) and induces AML in transgenic mice. Blood 2006, prepublished online June 8.

30 Hou YH, Srour EF, Ramsey H, et al. Identification of a human B-cell/myeloid common progenitor by the absence of CXCR4. Blood 2005;105:3488-3492.

31 Balciunaite G, Ceredig R, Massa S, et al. A $\mathrm{B} 220^{+} \mathrm{CD} 117^{+} \mathrm{CD} 19^{-}$hematopoietic progenitor with potent lymphoid and myeloid developmental potential. Eur J Immunol 2005;35:2019-2030.

32 Mckercher SR, Torbett BE, Anderson KL, et al. Targeted disruption of the PU.1 gene results in multiple hematopoietic abnormalities. EMBO J 1996;15: 5647-5658.

33 Rosenbauer F, Wagner K, Kutok JL, et al. Acute myeloid leukemia induced by graded reduction of a lineage-specific transcription factor, PU.1. Nat Genet 2004;36:624-630. 